\title{
$k$-RUN OVERPARTITIONS AND MOCK THETA FUNCTIONS
}

\author{
KATHRIN BRINGMANN, ALEXANDER E. HOLROYD, KARL MAHLBURG, AND MASHA VLASENKO
}

\begin{abstract}
In this paper we introduce $k$-run overpartitions as natural analogs to partitions without $k$-sequences, which were first defined and studied by Holroyd, Liggett, and Romik. Following their work as well as that of Andrews, we prove a number of results for $k$-run overpartitions, beginning with a double summation $q$-hypergeometric series representation for the generating functions. In the special case of 1-run overpartitions we further relate the generating function to one of Ramanujan's mock theta functions. Finally, we describe the relationship between $k$-run overpartitions and certain sequences of random events, and use probabilistic estimates in order to determine the asymptotic growth behavior of the number of $k$-run overpartitions of size $n$.
\end{abstract}

\section{INTRODUCTION AND STATEMENT OF RESUlts}

A partition of a positive integer $n$ is a non-increasing sequence of positive integers whose sum is $n$; the number of distinct partitions of $n$ is traditionally denoted by $p(n)$. A sequence (or run) in a partition is any subsequence of consecutive integers that appear as parts. Integer partitions without sequences were first studied by MacMahon in [21. He described their relationship to partitions with repeated parts, and also determined their generating series. These partitions were studied more recently by Holroyd, Liggett, and Romik in [17, where the authors introduced the general family of partitions without $k$-sequences for any $k \geq 2$, in which no $k$ consecutive integers may all appear as parts. The number of partitions of $n$ without $k$-sequences of $n$ is denoted by $p_{k}(n)$, and the generating function is defined as

$$
G_{k}(q):=\sum_{n \geq 0} p_{k}(n) q^{n}
$$

These partitions were also studied by Andrews [2], who found a (double) $q$-hypergeometric series expansion for the generating function. Before giving this series, we record the definition for the $q$-Pochhammer symbol, which is given by $(a ; q)_{n}:=\prod_{j=0}^{n-1}\left(1-a q^{j}\right)$.

Andrews showed that

$$
G_{k}(q)=\sum_{r, s \geq 0} \frac{(-1)^{r} q^{\frac{(k+1) k(r+s)^{2}}{2}}+\frac{(k+1)(s+1) s}{2}}{\left(q^{k} ; q^{k}\right)_{r}\left(q^{k+1} ; q^{k+1}\right)_{s}} .
$$

Both of these earlier papers also addressed the asymptotic behavior of $p_{k}(n)$ as $n \rightarrow \infty$. Holroyd, Liggett, and Romik [17] showed the asymptotic expression

$$
\log p_{k}(n) \sim \pi \sqrt{\frac{2}{3}\left(1-\frac{2}{k(k+1)}\right) n} \quad \text { as } n \rightarrow \infty .
$$

Date: November 12, 2018.

The research of the first author was supported by the Alfried Krupp Prize for Young University Teachers of the Krupp Foundation. The third author was supported by an NSF Postdoctoral Fellowship administered by the Mathematical Sciences Research Institute through its core grant DMS-0441170. The first and third authors were also partially supported by the Alexander von Humboldt Foundation. 
Andrews [2] substantially improved this result in the case $k=2$, proving the asymptotic expression

$$
p_{2}(n) \sim \frac{1}{4 \sqrt{3} n^{\frac{3}{4}}} e^{\frac{2 \pi}{3} \sqrt{n}} \text { as } n \rightarrow \infty .
$$

His proof relies on a beautiful and surprising relationship between $G_{2}(q)$ and one of Ramanujan's famous mock theta functions; we will further discuss this connection below.

Remark. The above asymptotic expression is not stated as such in Andrews' paper. He instead focused on the asymptotics of the generating series $G_{2}(q)$ as $q \rightarrow 1$. However, his results directly imply (1.3) upon applying a Tauberian theorem (cf. Section 4 of this paper). Recently (1.3) was greatly refined by the first and third authors of the present paper in [6]. Specifically, the latter paper introduced a generalization of the Circle Method in order to prove a series expansion for $p_{2}(n)$ involving Kloosterman sums, Bessel functions, and principal value integrals of modified Bessel functions.

In this paper we study a related family of overpartitions. As introduced by Corteel and Lovejoy in 12, an overpartition is a partition in which the last occurrence of each part may be overlined. The number of overpartitions of size $n$ is denoted by $\bar{p}(n)$. An overpartition is said to have a gap at $m$ if there are no parts of size $m$.

We define lower $k$-run overpartitions to be those overpartitions in which any overlined part must occur within a run of exactly $k$ consecutive overlined parts that terminates below with a gap. More precisely, this means that if some part $\bar{m}$ is overlined, then there is an integer $j$ with $m \in[j+1, j+k]$ such that each of the $k$ overlined parts $\varnothing j+1, \varnothing j+2, \ldots, \varnothing j+k$ appear (perhaps together with non-overlined versions), while no part $j$ (overlined or otherwise) appears, and no overlined part $\varnothing j+k+1$ appears. There is a simple bijection between lower $k$-run overpartitions and upper $k$-run overpartitions, which are defined analogously but with the conditions on parts $j$ and $j+k+1$ reversed (see Section 2.1). Denote the number of lower $k$-run overpartitions of size $n$ by $\bar{p}_{k}(n)$.

Example. The lower 2-run overpartitions of size 7 are

$$
\begin{gathered}
\varnothing 4+\varnothing 3, \quad 4+\varnothing 2+\varnothing 1, \quad \varnothing 3+2+\varnothing 2, \quad 3+\varnothing 2+1+\varnothing 1, \\
2+2+\varnothing 2+\varnothing 1, \quad 2+\varnothing 2+1+1+\varnothing 1, \quad \varnothing 2+1+1+1+1+\varnothing 1,
\end{gathered}
$$

together with the 15 partitions of 7 , so $\varnothing p_{2}(7)=7+15=22$.

The generating function for lower $k$-run overpartitions is denoted by

$$
\bar{G}_{k}(q):=\sum_{n \geq 0} \phi p_{k}(n) q^{n} .
$$

Our first result is analogous to Andrews' double-series generating function (1.1) for partitions without $k$-sequences.

Theorem 1.1. For $|q|<1$,

$$
\bar{G}_{k}(q)=\frac{1}{(q ; q)_{\infty}} \sum_{r, s \geq 0} \frac{(-1)^{s} q^{\frac{(k+1) k(r+s)^{2}}{2}}+\frac{(k+1) s(s+1)}{2}}{\left(q^{k} ; q^{k}\right)_{r}\left(q^{k+1} ; q^{k+1}\right)_{s}} .
$$

Our next result is an asymptotic expression for lower $k$-run overpartitions that is much stronger than the logarithmic expression in (1.2). 
Theorem 1.2. As $n \rightarrow \infty$,

$$
\bar{p}_{k}(n) \sim \frac{1}{2 \sqrt{6} n} \sqrt{1+\frac{1}{2 k(k+1)}} \exp \left(\pi \sqrt{\frac{2}{3}\left(1+\frac{1}{2 k(k+1)}\right) n}\right) .
$$

Remark. Interestingly, our techniques do not apply to the case of partitions without sequences, despite the similarity of (1.1) and Theorem 1.1.

We next focus particularly on certain special cases that are related to Ramanujan's mock theta functions. Andrews [2] showed that the generating function for partitions without 2-sequences may be written as

$$
G_{2}(q)=\frac{\left(-q^{3} ; q^{3}\right)_{\infty}}{\left(q^{2} ; q^{2}\right)_{\infty}} \cdot \chi(q)
$$

where

$$
\chi(q):=1+\sum_{n \geq 1} \frac{q^{n^{2}}}{\prod_{j=1}^{n}\left(1-q^{j}+q^{2 j}\right)},
$$

which is one of Ramanujan's third-order mock theta functions. Ramanujan originally introduced the mock theta functions by listing a small collection of examples in his last letter to Hardy [25]. He justified his own interest by observing their striking asymptotic properties and near-symmetries under modular transformations. Andrews [2] used some of these properties in order to determine the asymptotic behavior of $G_{2}(q)$ as $q \rightarrow 1$, which then implies (1.3). The general theory of mock theta functions has also recently seen great advancements, as Zwegers' doctoral thesis [28] has led to a proper understanding of the relationship to automorphic forms [9, 10, 26].

The case $k=1$ of lower $k$-run overpartitions is similarly related to another of Ramanujan's third-order mock theta functions from [25]. In particular, the mock theta function

$$
\phi(q):=1+\sum_{n \geq 1} \frac{q^{n^{2}}}{\left(-q^{2} ; q^{2}\right)_{n}},
$$

appears in the following expression for the generating function of lower 1-run overpartitions.

Theorem 1.3. For $|q|<1$,

$$
\bar{G}_{1}(q)=(q ; q)_{\infty} \cdot \phi(q) .
$$

Remark. Note that the $k=1$ case of Theorem 1.2 states that $\bar{p}_{1}(n) \sim \frac{\sqrt{5}}{4 \sqrt{6} n} e^{\pi \sqrt{\frac{5 n}{6}}}$.

The remainder of the paper is structured as follows. In Section 2, we consider basic combinatorial properties of $k$-run overpartitions and derive their generating series. In Section 3, we apply the Constant Term Method to determine the asymptotic behavior of the generating series. Finally, in Section 4, we prove the asymptotic expression for $k$-run overpartitions found in Theorem 1.2.

\section{OverPARTITION COMBINATORICS AND GENERATING SERIES}

2.1. Combinatorial results for $k$-run overpartitions. In this section we denote the number of lower $k$-run overpartitions of $n$ by $\bar{p}_{k}^{(-)}(n)$. We also recall the definition upper $k$-run overpartitions as overpartitions in which the overlined parts must occur in consecutive runs of the form $\varnothing j+1, \ldots, \overline{j+k}$, with no overlined parts $\varnothing j$, and no parts of any kind of size $j+k+1$. The number of upper $k$-run overpartitions of size $n$ is denoted by $\bar{p}_{k}^{(+)}(n)$.

Our first observation on $k$-run overpartitions is that the lower and upper definitions are in bijective correspondence. 
Proposition 2.1. For all $n \geq 0$ and $k \geq 1$,

$$
\bar{p}_{k}^{(-)}(n)=\bar{p}_{k}^{(+)}(n) .
$$

Proof. We construct a simple bijection between the two sets of overpartitions. In a lower $k$-run overpartition, any run of $k$ overlined parts occurs at the lower end of some block of consecutive parts, surrounded on both sides by gaps. That is, for some $j$ and some $\ell \geq j+k$, all the parts

$$
\varnothing j+1, \ldots, \varnothing j+k, j+k+1, \ldots, \ell
$$

appear (perhaps together with further non-overlined copies of these same values), with gaps at $j$ and $\ell+1$. We can form a new overpartition by shifting the $k$ overlines to the upper end of the block, i.e., replacing the above parts with

$$
j+1, \ldots, \ell-k, \varnothing \ell-k+1, \ldots, \varnothing \ell .
$$

Applying this transformation to every run of $k$ overlined parts results in an upper $k$-run overpartition, and this map is clearly bijective.

We henceforth write simply $\bar{p}_{k}$ for $\bar{p}_{k}^{(-)}$, and abbreviate the term "lower $k$-run overpartition" to $k$-run overpartition. It will be convenient to work with both the upper and lower definitions in different contexts. In view of Proposition 2.1 they are interchangeable for purposes of enumeration.

We have the following monotonicity properties for $k$-run overpartitions.

Proposition 2.2. For any $n \geq 0$ and $k \geq 1$,

(i) $\bar{p}_{k}(n) \leq \bar{p}_{k}(n+1)$

(ii) $\bar{p}_{k}(n) \geq \bar{p}_{k+1}(n)$.

Proof. ( $i$ Given an upper $k$-run overpartition of $n$, adding a non-overlined part 1 clearly gives an upper $k$-run overpartition of $n+1$, and this map is injective.

(ii) We construct an injection from lower $(k+1)$-run overpartitions to lower $k$-run overpartitions. If $\bar{\lambda}$ is a $(k+1)$-run overpartition and $\overline{j+1}, \ldots, \overline{j+k+1}$ is a $(k+1)$-run, then there are no parts of size $j$, and there can only be non-overlined parts of size $j+k+2$. Removing the overline on $\varnothing j+k+1$ results in a $k$-run satisfying the lower $k$-run condition, and applying this to every run gives the required injection.

\section{Remarks.}

(i) We note that the partitions without sequences studied in [17] satisfy the opposite inequality to that in Proposition $2.2($ ii $)$.

$$
p_{k}(n) \leq p_{k+1}(n) .
$$

(ii) For any fixed $n$, we have $\bar{p}_{k}(n)=p_{k}(n)=p(n)$ for sufficiently large $k$.

2.2. A double summation series. In this section we consider the two-variable generating functions

$$
\bar{G}_{k}(x)=\bar{G}_{k}(x ; q):=\sum_{\ell, n \geq 0} \bar{p}_{k}(\ell, n) x^{\ell} q^{n},
$$

where $\bar{p}_{k}(\ell, n)$ is defined to be the number of $k$-run overpartitions of size $n$ and exactly $\ell$ parts. The main result in this section is a double series expansion for this generating function, which is proven from a $q$-difference equation for $\bar{G}_{k}(x ; q)$. 
Theorem 2.3. For $|q|<1$,

$$
\bar{G}_{k}(x ; q)=\frac{1}{(x q ; q)_{\infty}} \sum_{r, s \geq 0} \frac{(-1)^{s} x^{k r+(k+1) s} q^{\frac{k(k+1)(r+s)^{2}}{2}}+\frac{(k+1) s(s+1)}{2}}{\left(q^{k} ; q^{k}\right)_{r}\left(q^{k+1} ; q^{k+1}\right)_{s}} .
$$

Proof. The generating function $\bar{G}_{k}$ satisfies the $q$-difference equation

$$
\bar{G}_{k}(x)=\frac{1}{1-x q} \bar{G}_{k}(x q)+\frac{x^{k} q^{\frac{k(k+1)}{2}}}{(x q ; q)_{k}} \bar{G}_{k}\left(x q^{k+1}\right),
$$

which follows from the definition of (upper) $k$-run overpartitions by separating according to their smallest parts. The first term corresponds to the overpartitions without an overlined $\overline{1}$, in which case there is no restriction on subsequent parts; the second term corresponds to overpartitions that do have the part $\overline{1}$, and thus also have each of the parts $\overline{2}, \ldots, \bar{k}$ (and possibly non-overlined parts of these sizes as well), followed by no parts of size $k+1$.

This recurrence is easier to solve after "re-normalizing" the equation, which is achieved by setting

$$
\bar{L}_{k}(x)=\bar{L}_{k}(x ; q):=(x q ; q)_{\infty} \bar{G}_{k}(x ; q) .
$$

Equation (2.2) then implies that this normalized function satisfies the $q$-difference equation

$$
\bar{L}_{k}(x)-\bar{L}_{k}(x q)=x^{k} q^{\frac{k(k+1)}{2}}\left(1-x q^{k+1}\right) \bar{L}_{k}\left(x q^{k+1}\right) .
$$

We next write this as a series in $x$, where the coefficients are polynomials in $q, \bar{L}_{k}(x ; q)=$ : $\sum_{m \geq 0} \bar{\lambda}_{m}(q) x^{m}$. Equation (2.4) is equivalent to the statement that for all $m$,

$$
\left(1-q^{m}\right) \bar{\lambda}_{m}=q^{m(k+1)-\frac{k(k+1)}{2}}\left(\bar{\lambda}_{m-k}-\bar{\lambda}_{m-k-1}\right) .
$$

By (2.3), the theorem statement is equivalent to verifying that $\bar{L}_{k}(x ; q)$ is equal to the double series

$$
\sum_{r, s \geq 0} \frac{(-1)^{s} x^{k r+(k+1) s} q^{\frac{k(k+1)(r+s)^{2}}{2}}+\frac{(k+1) s(s+1)}{2}}{\left(q^{k} ; q^{k}\right)_{r}\left(q^{k+1} ; q^{k+1}\right)_{s}} .
$$

It is sufficient to show that this double series satisfies the $q$-difference equation (2.4), while also checking that the $k+1$ initial $x$-powers are compatible. Denote the $x^{m}$ coefficient in the double series by $\widetilde{\lambda}_{m}$; it is clear by definition that $\widetilde{\lambda}_{0}=1$ and that $\widetilde{\lambda}_{1}=\cdots=\widetilde{\lambda}_{k-1}=0$, and finally

$$
\widetilde{\lambda}_{k}=\frac{x^{k} q^{\frac{k(k+1)}{2}}}{1-q^{k}} \text {. }
$$

Note that $k$-run overpartitions with at most $k-1$ parts can contain only non-overlined parts, and thus

$$
\bar{G}_{k}(x ; q)=\frac{1}{(x q ; q)_{\infty}}+\frac{x^{k} q^{\frac{k(k+1)}{2}}}{1-q^{k}}+O\left(x^{k+1}\right) .
$$

The first term gives all overpartitions with no overlined parts, and the second term gives all overpartitions that consist of a single $k$-run of overlined parts. Thus

$$
\bar{L}_{k}(x ; q)=1+\frac{x^{k} q^{\frac{k(k+1)}{2}}}{1-q^{k}}+O\left(x^{k+1}\right),
$$

and the initial terms $\lambda_{i}(0 \leq i \leq k)$ agree with those given for $\widetilde{\lambda}_{i}$ above. 
Now we verify that the $\widetilde{\lambda}_{i}$ satisfy (2.5). Temporarily writing

$$
A_{r, s}=A_{r, s}(q):=\frac{(-1)^{s} q^{\frac{k(k+1)(r+s)^{2}}{2}}+\frac{(k+1) s(s+1)}{2}}{\left(q^{k} ; q^{k}\right)_{r}\left(q^{k+1} ; q^{k+1}\right)_{s}}
$$

we then have $\widetilde{\lambda}_{m}=\sum_{k r+(k+1) s=m} A_{r, s}$, and thus

$$
\begin{aligned}
\left(1-q^{m}\right) \widetilde{\lambda}_{m}= & \sum_{\substack{r, s \geq 0: \\
k r+(k+1) s=m}} A_{r, s} \cdot\left[1-q^{(k+1) s}+q^{(k+1) s}\left(1-q^{k r}\right)\right] \\
= & \sum_{\substack{r, s \geq 0: \\
k r+(k+1) s=m-k-1}} A_{r, s} \cdot q^{\frac{k(k+1)(2 r+2 s+1)}{2}+(k+1)(s+1)} \\
& \quad+\sum_{\substack{r, s \geq 0: \\
k r+(k+1) s=m-k}} A_{r, s} \cdot q^{\frac{k(k+1)(2 r+2 s+1)}{2}+(k+1) s} \\
= & q^{m(k+1)-\frac{k(k+1)}{2}}\left(\widetilde{\lambda}_{m-k}-\widetilde{\lambda}_{m-k-1}\right) .
\end{aligned}
$$

The two sums in the second line were shifted by $s \mapsto s+1$ and $r \mapsto r+1$, respectively. This completes the proof of the double series formula.

2.3. Relation to Ramanujan's mock theta functions. In this section we prove Theorem 1.3 . Recall the earlier definition of Ramanujan's third-order mock theta function

$$
\phi(q):=\sum_{n \geq 0} \frac{q^{n^{2}}}{\left(-q^{2} ; q^{2}\right)_{n}} .
$$

This was proven by Fine (see equation (26.32) of [15]) to have the equivalent form

$$
\phi(q)=\frac{1}{(q ; q)_{\infty}}\left(1+2 \sum_{n \geq 1} \frac{q^{n}}{1-q^{n}} \prod_{j=1}^{n-1} \frac{1+q^{2 j}}{1-q^{j}}\right) .
$$

Proof of Theorem 1.3. We first describe a family of overpartitions that are in bijective correspondence with lower 1-run overpartitions. The bijection will be the conjugation involution. An overpartition may be represented by a Ferrer's diagram that lists each part as a left-justified row of dots, with the parts listed in decreasing order from top to bottom. Furthermore, since an overlined part is the last occurrence of any part size, we distinguish it by marking the corresponding bottom-right "corner". The conjugation map then simply interchanges the rows and columns of such a diagram.

Under overpartition conjugation, lower 1-run overpartitions map to overpartitions in which overlined parts may not occur alone, except for possibly in the largest part. In other words, such overpartitions have the property that if $\bar{m} \in \lambda$ and $m$ is not the largest part size, then $m \in \lambda$ as well. See Figure 1 for an example of the conjugate of a 1-run overpartition.

By distinguishing the largest part, the generating function for such partitions is clearly

$$
\bar{G}_{1}(q)=1+2 \sum_{n \geq 1}\left(q^{n}+q^{2 n}+\cdots\right) \prod_{j=1}^{n-1}\left(1+q^{j}+2 q^{2 j}+2 q^{3 j}+\cdots\right),
$$

which is easily seen to be equal to the second factor in (2.6). 


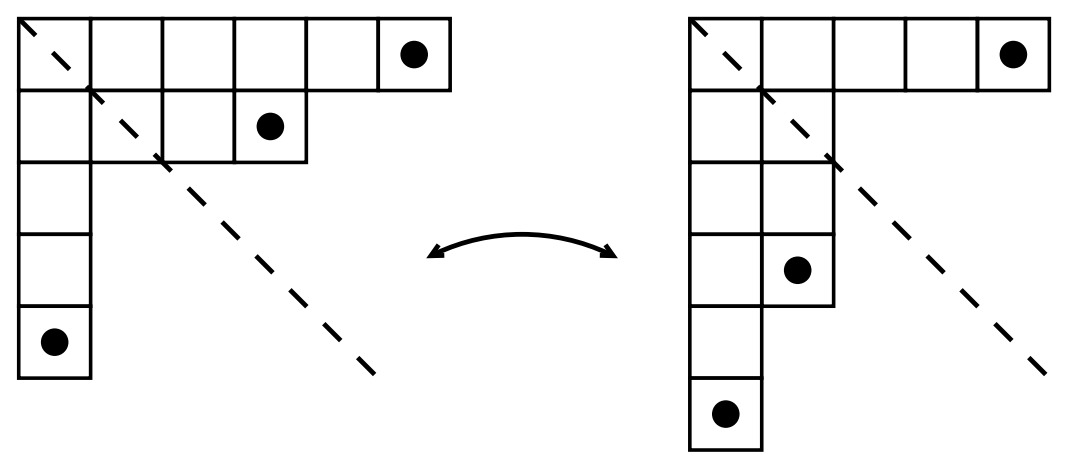

Figure 1. The overpartition on the left, $\overline{6}+\overline{4}+1+1+\overline{1}$, is a lower 1-run overpartition. On the right is its conjugate, $\overline{5}+2+2+\overline{2}+1+\overline{1}$, which has the property that (with the exception of the largest part), no overlined part size occurs alone.

\section{Generating series and the Constant Term Method}

Throughout this section we view $q$-series as Fourier expansions. In fact, we will mainly focus on real values of $q \in(0,1)$, so we write $q:=e^{-\varepsilon}$ with $\varepsilon \in \mathbb{R}^{+}$. Consider the double hypergeometric $q$-series from Theorem 1.1, which we denote as

$$
H_{k}(q):=\sum_{r, s \geq 0} \frac{(-1)^{s} q^{\frac{k(k+1)(r+s)^{2}}{2}}+\frac{(k+1) s(s+1)}{2}}{\left(q^{k} ; q^{k}\right)_{r}\left(q^{k+1} ; q^{k+1}\right)_{s}}=(q ; q)_{\infty} \bar{G}_{k}(q) .
$$

Proposition 3.1. As $\varepsilon \rightarrow 0^{+}$,

$$
H_{k}(q)=\sqrt{2} e^{\frac{\pi^{2}}{12 k(k+1) \varepsilon}}\left(1+O\left(\varepsilon^{\frac{1}{2}}\right)\right) .
$$

Proof. The proof follows the constant-term method, which begins with the observation that $H_{k}$ can be written as a (Laurent) coefficient of a two-variable series, namely

$$
H_{k}(q)=\text { coeff }\left[x^{0}\right]\left(\sum_{n \in \mathbb{Z}} q^{\frac{k(k+1) n^{2}}{2}} x^{-n} \sum_{r \geq 0} \frac{x^{r}}{\left(q^{k} ; q^{k}\right)_{r}} \sum_{s \geq 0} \frac{(-1)^{s} x^{s} q^{\frac{(k+1) s(s+1)}{2}}}{\left(q^{k+1} ; q^{k+1}\right)_{s}}\right) .
$$

As indicated, the series on the right side of (3.1) factorizes into three sums (in $n, r$, and $s$ ), and we now express each of the three factors in terms of known number-theoretic functions. Note that all of the summations are convergent for $|x|,|q|<1$. The first factor is $\theta\left(q^{\frac{k(k+1)}{2}} ; x\right)$, where

$$
\theta(q ; x):=\sum_{n \in \mathbb{Z}} q^{n^{2}} x^{n}
$$

is the Jacobi theta function. 
The second and third summations can both be expressed as infinite products via the following instances of the $q$-binomial theorem [3]:

$$
\begin{aligned}
\frac{1}{(x ; q)_{\infty}} & =\sum_{m \geq 0} \frac{x^{m}}{(q ; q)_{m}}, \\
(x ; q)_{\infty} & =\sum_{m \geq 0} \frac{(-1)^{m} q^{\frac{m(m-1)}{2}} x^{m}}{(q ; q)_{m}} .
\end{aligned}
$$

We further rewrite these terms using the quantum dilogarithm; this function is defined by

$$
\operatorname{Li}_{2}(x ; q):=-\log (x ; q)_{\infty}=\sum_{m \geq 1} \frac{x^{m}}{m\left(1-q^{m}\right)} .
$$

The expression in (3.1) can now be written as

$$
H_{k}(q)=\operatorname{coeff}\left[x^{0}\right]\left(\theta\left(q^{\frac{k(k+1)}{2}} ; x\right) \exp \left(\operatorname{Li}_{2}\left(x ; q^{k}\right)-\operatorname{Li}_{2}\left(x q^{k+1} ; q^{k+1}\right)\right)\right) .
$$

Applying Cauchy's theorem, we recover the $x^{0}$-coefficient as

$$
H_{k}(q)=\int_{[0,1]+i c} \theta\left(q^{\frac{k(k+1)}{2}} ; e^{2 \pi i u}\right) \exp \left(\operatorname{Li}_{2}\left(e^{2 \pi i u} ; q^{k}\right)-\operatorname{Li}_{2}\left(e^{2 \pi i u} q^{k+1} ; q^{k+1}\right)\right) d u,
$$

where $c>0$ is a real constant that will be specified later. The theta function may be transformed using the standard Poisson summation formula [19], resulting in

$$
\theta\left(e^{-\frac{k(k+1) \varepsilon}{2}} ; e^{2 \pi i u}\right)=\sum_{n \in \mathbb{Z}} e^{\frac{-k(k+1) n^{2} \varepsilon}{2}-2 \pi i n u}=\sqrt{\frac{2 \pi}{\varepsilon k(k+1)}} \sum_{n \in \mathbb{Z}} e^{-\frac{2 \pi^{2}(n+u)^{2}}{\varepsilon k(k+1)}} .
$$

Our integral then transforms to

$$
\begin{aligned}
H_{k}(q) & =\sqrt{\frac{2 \pi}{\varepsilon k(k+1)}} \int_{[0,1]+i c} \sum_{n \in \mathbb{Z}} \exp \left(-\frac{2 \pi^{2}(n+u)^{2}}{\varepsilon k(k+1)}+\operatorname{Li}_{2}\left(e^{2 \pi i u} ; q^{k}\right)-\operatorname{Li}_{2}\left(e^{2 \pi i u} q^{k+1} ; q^{k+1}\right)\right) d u \\
& =\sqrt{\frac{2 \pi^{2}}{\varepsilon k(k+1)}} \int_{\mathbb{R}+i c} \exp \left(-\frac{2 \pi u^{2}}{\varepsilon k(k+1)}+\operatorname{Li}_{2}\left(e^{2 \pi i u} ; e^{-k \varepsilon}\right)-\operatorname{Li}_{2}\left(e^{2 \pi i u-(k+1) \varepsilon} ; e^{-(k+1) \varepsilon}\right)\right) d u .
\end{aligned}
$$

We now apply the stationary phase method to this integral, which is generally useful for determining the asymptotic behavior of integrals of the form $\int_{\mathbb{R}} g(u) e^{\frac{i f(u)}{\varepsilon}} d u$ as $\varepsilon \rightarrow 0^{+}$. If $f$ has a critical point at $u_{0}$, and both $f$ and $g$ have Taylor expansions around this point, then the dominant asymptotic term of the integral can be described in terms of $f\left(u_{0}\right)$ and $g\left(u_{0}\right)$.

In order to apply the stationary phase method to (3.2), we must first rewrite the integrand in this shape in order to identify the dominant asymptotic terms. If $|x|<1$ and $B \geq 0$, then the Laurent expansion of the quantum dilogarithm begins

$$
\begin{aligned}
\operatorname{Li}_{2}\left(e^{-B \varepsilon} x ; e^{-\varepsilon}\right) & =\sum_{n \geq 1} \frac{x^{n} e^{-B n \varepsilon}}{n\left(1-e^{-\varepsilon n}\right)}=\frac{1}{\varepsilon} \sum_{n \geq 1} \frac{x^{n}}{n^{2}}\left(1-n \varepsilon\left(B-\frac{1}{2}\right)+O\left(\varepsilon^{2}\right)\right) \\
& =\frac{1}{\varepsilon} \operatorname{Li}_{2}(x)+\left(B-\frac{1}{2}\right) \log (1-x)+O(\varepsilon)
\end{aligned}
$$


uniformly in $x$ as $\varepsilon \rightarrow 0$. Here $\operatorname{Li}_{2}(x):=\sum_{n>1} \frac{x^{n}}{n^{2}}$ is the standard dilogarithm function. Using (3.3), we study the argument of the exponential in the integrand of (3.2), namely

$$
-\frac{2 \pi u^{2}}{\varepsilon k(k+1)}+\operatorname{Li}_{2}\left(e^{2 \pi i u} ; e^{-k \varepsilon}\right)-\operatorname{Li}_{2}\left(e^{2 \pi i u-(k+1) \varepsilon} ; e^{-(k+1) \varepsilon}\right) .
$$

In particular, we consider the Laurent expansion of (3.4) and denote the coefficient of the (leading) $\varepsilon^{-1}$ term by

$$
f(u):=-\frac{2 \pi^{2} u^{2}}{k(k+1)}-\frac{\operatorname{Li}_{2}\left(e^{2 \pi i u}\right)}{(k+1)}+\frac{\operatorname{Li}_{2}\left(e^{2 \pi i u}\right)}{k}=-\frac{2 \pi^{2} u^{2}}{k(k+1)}+\frac{\operatorname{Li}_{2}\left(e^{2 \pi i u}\right)}{k(k+1)} .
$$

We proceed by determining the critical point(s) of $f$. Its derivative is

$$
f^{\prime}(u)=-\frac{4 \pi^{2} u}{k(k+1)}+\frac{2 \pi i \log \left(1-e^{2 \pi i u}\right)}{k(k+1)}=\frac{2 \pi i}{k(k+1)}\left(2 \pi i u-\log \left(1-e^{2 \pi i u}\right)\right),
$$

and the critical point therefore occurs at

$$
e^{2 \pi i u}=1-e^{2 \pi i u}, \quad \text { i.e., } \quad u=\frac{i \log 2}{2 \pi} .
$$

We denote this critical value of $u$ by $w:=\frac{i \log 2}{2 \pi}$, which also determines the most appropriate height of the contour (specifically, $c=\frac{\log 2}{2 \pi}$ ). At the critical value, the function $f$ evaluates to

$$
f(w)=\frac{(\log 2)^{2}}{2 k(k+1)}+\frac{1}{k(k+1)} \operatorname{Li}_{2}\left(\frac{1}{2}\right)=\frac{\pi^{2}}{12 k(k+1)} .
$$

Here we used the functional equation [27]

$$
\mathrm{Li}_{2}(x)+\mathrm{Li}_{2}(1-x)=\frac{\pi^{2}}{6}-\log (x) \log (1-x) .
$$

By definition $f^{\prime}(w)=0$, but the second derivative still makes a contribution to the overall asymptotic behavior, so we calculate

$$
f^{\prime \prime}(w)=\frac{2 \pi i}{k(k+1)}\left(2 \pi i+\frac{2 \pi i e^{2 \pi i u}}{1-e^{2 \pi i u}}\right)=-\frac{8 \pi^{2}}{k(k+1)}<0 .
$$

We can therefore compute the first terms in the Taylor expansion around $w$ of (3.4). In particular, using the change of variable $u=w+\sqrt{\varepsilon} z$, this Taylor expansion is

$$
\frac{f(w)}{\varepsilon}+\left(\frac{f^{\prime \prime}(w)}{2} z^{2}-\log \left(1-e^{2 \pi i w}\right)\right)+O\left(\varepsilon^{\frac{1}{2}}\right) .
$$

Plugging in the expansions (3.3) and (3.5) to the integral (3.2), the contour may then be shifted to the real axis, leading finally to the evaluation

$$
\begin{aligned}
H_{k}(q) & =\sqrt{\frac{2 \pi}{\varepsilon k(k+1)}} \frac{e^{\frac{f(w)}{\varepsilon}}}{1-e^{2 \pi i w}}\left(1+O\left(\varepsilon^{\frac{1}{2}}\right)\right) \int_{\mathbb{R}} e^{\frac{f^{\prime \prime}(w) x^{2}}{2 \varepsilon}} d x \\
& =\sqrt{\frac{2 \pi}{\varepsilon k(k+1)}} e^{\frac{\pi^{2}}{12 k(k+1) \varepsilon}} \cdot 2 \cdot \sqrt{\frac{2 \varepsilon}{-f^{\prime \prime}(w)}} \sqrt{\pi}\left(1+O\left(\varepsilon^{\frac{1}{2}}\right)\right) \\
& =\sqrt{2} e^{\frac{\pi^{2}}{12 k(k+1) \varepsilon}}\left(1+O\left(\varepsilon^{\frac{1}{2}}\right)\right) .
\end{aligned}
$$




\section{Asymptotic Behavior of $\bar{p}_{k}(n)$}

In this section we use the asymptotic behavior of generating series in order to determine the asymptotic behavior of $\bar{p}_{k}(n)$ as $n \rightarrow \infty$. As before, we write $q=e^{-\varepsilon}$. Recall that

$$
\bar{G}_{k}(q)=\sum_{n=0}^{\infty} \bar{p}_{k}(n) q^{n}=(q ; q)_{\infty}^{-1} \cdot H_{k}(q) .
$$

Ingham's Tauberian theorem relates the asymptotic behavior of such a series to its coefficients. The following result is a special case of Theorem 1 in [18].

Theorem 4.1 (Ingham). Let $f(z)=\sum_{n>0} a(n) z^{n}$ be a power series with real nonnegative coefficients and radius of convergence equal to 1 . If there exist $A>0, \lambda, \alpha \in \mathbb{R}$ such that

$$
f(z) \sim \lambda(-\log z)^{\alpha} \exp \left(\frac{A}{-\log z}\right)
$$

as $z \rightarrow 1^{-}$, then

$$
\sum_{m=0}^{n} a(m) \sim \frac{\lambda}{2 \sqrt{\pi}} \frac{A^{\frac{\alpha}{2}-\frac{1}{4}}}{n^{\frac{\alpha}{2}+\frac{1}{4}}} \exp (2 \sqrt{A n})
$$

as $n \rightarrow \infty$.

Proof of Theorem 1.2. The modular inversion formula for Dedekind's eta-function (page 121, Proposition 14 of [19]) states that

$$
(q ; q)_{\infty}=\sqrt{\frac{2 \pi}{\varepsilon}} e^{-\frac{\varepsilon}{24}-\frac{\pi^{2}}{6 \varepsilon}} \prod_{n \geq 1}\left(1-e^{-\frac{4 \pi^{2} n}{\varepsilon}}\right) .
$$

This implies that $\varepsilon \rightarrow 0^{+}$,

$$
(q ; q)_{\infty} \sim \sqrt{\frac{2 \pi}{\varepsilon}} e^{-\frac{\pi^{2}}{6 \varepsilon}}
$$

Combined with Proposition 3.1, this implies that as $\varepsilon \rightarrow 0^{+}$

$$
(1-q) \bar{G}_{k}(q) \sim \frac{\sqrt{\varepsilon}}{\sqrt{\pi}} \exp \left(\frac{\pi^{2}}{6 \varepsilon}\left(1+\frac{1}{2 k(k+1)}\right)\right) .
$$

Note that the coefficients of this $q$-series are $(1-q) \bar{G}_{k}(q)=\sum_{n>0}\left(\bar{p}_{k}(n)-\bar{p}_{k}(n-1)\right) q^{n}$. Applying Theorem 4.1 with $a(n):=\bar{p}_{k}(n)-\bar{p}_{k}(n-1)$ then gives the stated asymptotic formula for $\bar{p}_{k}(n)$ by telescoping.

\section{REFERENCES}

[1] G. Andrews, A survey of multipartitions: Congruences and identities, Surveys in Number Theory, K. Alladi, ed., Developments in Math. 17, 1-19, Springer, 2008.

[2] G. Andrews, Partitions with short sequences and mock theta functions, Proc. Nat. Acad. Sci. 102 (2005), 46664671.

[3] G. Andrews, The theory of partitions, Cambridge University Press, Cambridge, 1998.

[4] G. Andrews, R. Askey, and R. Roy, Special functions, Cambridge University Press, Cambridge, 1999.

[5] G. Andrews, H. Eriksson, F. Petrov, and D. Romik, Integrals, partitions and MacMahon's theorem, J. Comb. Theory (A) 114 (2007), 545-554.

[6] K. Bringmann and K. Mahlburg, An extension of the Hardy-Ramanujan circle method and applications to partitions without sequences, American J. of Math. 133 (2011), 1151-1178.

[7] K. Bringmann, K. Mahlburg, Improved bounds on metastability thresholds and probabilities for generalized bootstrap percolation, Transactions of the AMS, accepted for publication. 
[8] K. Bringmann, K. Mahlburg, and A. Mellit, Convolution bootstrap percolation models, Markov-type stochastic processes, and mock theta functions, submitted for publication.

[9] K. Bringmann and K. Ono, Coefficients of harmonic weak Maass forms, Proc. of the 2008 Univ. of Florida Conf. on $q$-series, partitions, and modular forms.

[10] K. Bringmann and K. Ono, Dyson's ranks and Maass forms, Ann. of Math. (2) 171 (2010), 419-449.

[11] J. Bruinier and J. Funke, On two geometric theta lifts, Duke Math. Journal 125 (2004), 45-90.

[12] S. Corteel and J. Lovejoy, Overpartitions, Trans. Amer. Math. Soc. 356 (2004), 1623-35.

[13] L. Dragonette, Some asymptotic formulae for the mock theta series of Ramanujan, Trans. Amer. Math. Soc. 72 (1952), 474-500.

[14] L. Faddeev and R. Kashaev, Quantum dilogarithm, Modern Phys. Lett. A 9 (1994), 427-434.

[15] N. Fine, Basic hypergeometric series and applications, Amer. Math. Soc., Providence, RI, 1988.

[16] G. Hardy and S. Ramanujan, Asmptotic formulae for the distribution of integers of various types, Proc. London Math. Soc. (2) 16 (1918), 112-32.

[17] A. Holroyd, T. Liggett, and D. Romik, Integrals, partitions, and cellular automata, Trans. Amer. Math. Soc. 356 (2004), 3349-3368.

[18] A. Ingham, A Tauberian theorem for partitions, Ann. of Math. 42 (1941), 1075-1090.

[19] N. Koblitz, Introduction to elliptic curves and modular forms, Graduate Texts in Mathematics 97, SpringerVerlag, New York, 1984.

[20] M. Koecher and A. Krieg, Elliptische funktionen und modulformen, second ed., Springer-Verlag, Berlin, 2007.

[21] P. MacMahon, Combinatory Analysis Vol. II, Cambridge Univ. Press, Cambridge, 1916, 49-58.

[22] K. Ono, The Web of Modularity, CBMS Regional Conference Series in Math. 102 (American Mathematical Society), 2004.

[23] H. Rademacher, On the expansion of the partition function in a series, Ann. of Math. (2) 44, (1943), 416-422.

[24] H. Rademacher and H. S. Zuckerman, On the Fourier coefficients of certain modular forms of positive dimension, Ann. of Math. (2) 39 (1938), no. 2, pages 433-462.

[25] G. Watson, The final problem: An account of the mock theta functions, J. London Math. Soc. 11 (1936), 55-80.

[26] D. Zagier, Ramanujan's mock theta functions and their applications [d'aprés Zwegers and Bringmann-Ono] (2006), Séminaire Bourbaki, no 986.

[27] D. Zagier, The dilogarithm function, Frontiers in number theory, physics, and geometry II, 3-65, Springer, Berlin, 2007.

[28] S. Zwegers, Mock theta functions, Ph.D. Thesis, Universiteit Utrecht, 2002.

Mathematical Institute, University of Cologne, Weyertal 86-90, 50931 Cologne, Germany

E-mail address: kbringma@math.uni-koeln.de

Microsoft Research, 1 Microsoft Way, Redmond, WA 98052, USA

E-mail address: holroyd at microsoft.com

Department of Mathematics, Princeton University, NJ 08544, U.S.A.

E-mail address: mahlburg@math.princeton.edu

E-mail address: masha.vlasenko@gmail.com 